HOW TO...

\title{
Write a Case Report
}

\author{
I Greaves
}

\begin{abstract}
Case reports are often seen as a relatively easy way of getting published. Despite this, much wasted time and effort on the part of authors, reviewers and editors might be saved if intending authors followed some simple guidelines before committing themselves to paper. The rejection rate for case reports is extremely high. There are two main reasons for this: inappropriate case selection and inadequate attention to detail in case preparation. This article aims to offer a template to the clinician who is considering writing a case report, in order to make the most efficient use (in terms of published results) of the time available.
\end{abstract}

\section{Introduction}

Although individual case reports are often highly memorable, such reports are often considered to be a second rate way of getting published. There can be little doubt that at least part of this unfavourable attitude is due to the poor quality of many published reports. It is also true to say that increasing numbers of case reports are usually seen as a sign of a journal which is failing. The fewer case reports a journal publishes, the more prestigious an acceptance for the authors. Nevertheless, case reports are an important category of publication and with appropriate case selection and considerable attention to detail in preparation they are a powerful way of highlighting clinical topics, reminding clinicians of rare but important conditions and identifying a need to change clinical practice. Unfortunately, many authors, especially those who are relatively inexperienced or new to publishing, waste a great deal of time in the preparation of reports which are highly unlikely to be accepted by a reputable academic journal. Although case reports undoubtedly involve less work than clinical trials, treating them as an easy option only produces slapdash results and reduces the chances of success.

Maj Ian Greaves RAMC

HQ AMS,

FASC, Slim Road, Camberley,

Surrey, GU15 4NP

\section{Why write a case report? (see Table 1)}

There are a number of potential reasons for writing a case report. Whichever apply, it is worth remembering that all publications are for the primary benefit of the reader, not the author.

\section{Write for the readers not for the author}

The first (although not necessarily the best) reason for writing a case report is to increase the number of one's publications (the "never mind the quality feel the width" syndrome). In today's highly competitive world, this has to be considered a legitimate reason for venturing into print, especially for junior clinicians when the constraints of changing job every six months are a serious handicap to more ambitious projects.

The second reason is to educate the reader, either about a rare condition, or about an unusual aspect of a more common one. A subgroup includes those case reports which are designed to change clinical practice: when you see this presentation, think about this (unusual) diagnosis or consider this method of treatment. ("We didn't, and look what happened!')

It is also perfectly legitimate to write a case report in order to amuse (the Christmas $B M 7$ approach) although there are one or two furrows (usually involving foreign bodies) which have probably been over ploughed. The reactions of one's colleagues are a useful guide to the suitability of potential cases.

Table 1. Reasons for writing a case report

- achieving a publication

- to educate

- to change practice

- to amuse

\section{Writing a case report}

Keeping a list

The initial step is to have the idea! It is always useful to keep a list of interesting cases in a notebook carried in the pocket or pinned to a wall in the office. Sufficient data to allow the patient records to be obtained must be recorded at the time. Attempting to obtain patient details retrospectively is rarely successful. In addition, written 
consent from the patient to publication should be sought at this stage and kept in a safe place. Approaching the patient for consent at a later stage is inconvenient and may not be possible. It is often helpful to make notes at the time and keep these (in a secure place) since the hospital notes may not be available at a later date. Radiographic images should be copied or photographed.

Keeping a list of patients also allows short series of patients with similar presentations to be identified and stops conversations along the lines of "We had another patient just like this a few months ago, can you remember his name?". A list also allows the most promising case for publication to be selected for writing up from a shortlist of potential cases.

\section{Selecting a case}

The most appropriate response in the majority of cases to the question: "Is this case worth writing up?" is a resounding "No!" Selection of an appropriate case is essential if wasted effort is to be avoided. The ability to make a choice from a list of interesting cases is a significant advantage and sharing interesting cases with colleagues, which can be written up jointly, is useful. Any case should be discussed with one or more senior or experienced colleagues, who will be able to advise about suitability for publication, before work commences in earnest. A careful literature search is also essential in order to ensure that similar cases have not been recently published. If there is any doubt, it is better to abandon a case rather than waste hours of effort which is very unlikely to be rewarded.

There are a number of types of case which are very much better avoided (Table 2 ).

Clearly, everyone's ambition is to write up the first case of a completely new condition and achieve eponymous fame. In practice, such cases are extremely rare, and very unusual cases are usually the best that can be hoped for. In this case, some judgment is called for. Whilst it is undoubtedly worth reporting the second case of a rare condition, reporting the thirty ninth case is unlikely to be of any value. However, reporting a new case of a rare condition can be enhanced in two ways, either by including a review of the previous literature, or, by reporting a short series of new cases. These options will be discussed below. A report of a "classic case" of an extraordinarily rare, but well recognized condition serves little purpose and is unlikely to be accepted.

Despite the medical profession's enduring enthusiasm for freaks and foreign bodies, both tend to be overused as subjects for case reports. Common conditions or objects do not necessarily become fascinating simply because they occur in unusual places. Cases along the lines of endometriosis in the left external auditory meatus are best avoided.

Table 2. Bad ideas

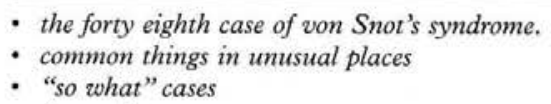

- the forty eighth case of von Snot's syndrome.

- common things in unusual places

- "so what" cases

- incomplete cases

If a case is to be published, it is essential that all the relevant results, documentation, investigations and follow up are available. Incomplete or doubtful cases are unlikely to be accepted.

The "so what" test should be applied to any potential case. Does the paper have a message? More importantly, would you, the author, want to read it if someone else had written it? If the answer is no, it is highly unlikely that any one else would either.

\section{Would you want to read it?}

\section{Selecting a journal}

Editors of journals are often surprised when completely inappropriate material, which has often (but sadly not always) involved a great deal of hard labour is submitted to them. In order to avoid the disappointment of repeated rejections, it is vital that a suitable journal is selected and that the paper is carefully tailored to the relevant "house style". The most prestigious journal which is suitable should be chosen as the first potential recipient of the paper. The style, presentation, and indeed the quality of published case reports varies dramatically from journal to journal. Reading and complying with the instructions for authors is mandatory.

\section{Read and comply with the instructions for authors}

A careful study of recent case reports from the journal which is finally chosen will provide essential information regarding length, style, referencing and illustrations. If clinical photographs are to be included, written permission will be required from the patient.

\section{Submitting the report}

Before the report is submitted, it should be read by several people, not only to check its medical content but to eliminate spelling and syntactical errors. It is useful if one of these readers is is non-medical as they often spot errors that are missed by doctors, especially typographical ones like the extra is earlier in this sentence. Authors tend to see only what they expect to see. However startling the content, if the English is not fluent and the impression is of a hurried careless submission, publication is unlikely. It is not the job of a journal editor to 
transform confused ramblings in what appears to be the author's second language into breathless lyrical prose. Word count limits must be strictly adhered to.

The report should be submitted in exactly the form specified in the instructions for authors. If the editor requires the illustrations and tables to be on separate sheets with the captions also submitted and labeled individually, this instruction must be followed. It is important not to write on the back of photographs since imprints of writing may effect the quality of the image as it appears in the journal. Increasingly, journals will request an electronic copy of the report on disc. Although this is rarely mandatory, it makes the task of the editor easier and gives an impression of efficiency. The specified format and word processing package must be used. Some journals accept illustrations on disc but if this is not stated in the instructions for authors, conventional images should be sent. Photographs may be either in colour or black and white and may be submitted as slides or prints. The requested number of copies must be included. A covering letter signed by all the authors offering the article for publication must also included. Instructions for authors sometimes require specific declarations (usually that the article is not being submitted elsewhere) and these should be included as appropriate.

\section{Increasing the chances of getting published}

The best case reports have a clear message. In order to ensure that this is emphasized in the finished paper, it is important that the authors are clear in their own minds about precisely what message they are intending to convey. Unless the report is intended primarily to amuse, if there is no message, the report is probably not worth submitting for publication.

Assuming the paper is considered, after consultation with colleagues, to be worthy of writing up, inclusion of a review of the relevant literature (within the wordcount $\stackrel{c}{\complement}$ and format specified by the journal) will $D$ increase the attractiveness of the paper. Carefully chosen, high quality illustrations $z$ (usually in limited numbers) should also be $\stackrel{\varnothing}{\Omega}$ included, remembering that in the majority $?$ of cases they will need to show whatever they are intended to show clearly in black. and white. This is most often a problem with illustrations of skin conditions.

\section{Photographic Cases}

Some journals publish short reports $\stackrel{\mathbb{Q}}{\complement}$ consisting of a photograph and a short accompanying text (usually a single $\overrightarrow{0}$ paragraph). The most well known of these is $\vec{\overrightarrow{ }}$ "Minerva" in the British Medical Journal. Such photographs provide an excellent minimal effort way of getting into print. Now that digital cameras are available, any aspiring clinician should have access to one, saving the inconvenience of having to send $\underset{\omega}{\dot{\omega}}$ patients to medical illustration. Written $\stackrel{\dot{V}}{V}$ consent to publish must be sought from the $\mathrm{o}$ patient or guardian (in the case of a child). Once a photograph has been chosen for $O$ submission, considerable care should be $\frac{\mathcal{f}}{0}$ taken in preparing the accompanying text.

\section{Buy a digital camera -now}

\section{Conclusion}

Case reports are often undervalued. They are, however, an important part of the medical literature and can be highly $\mathbb{\Phi}$ effective in changing clinical practice. Much $\overrightarrow{\vec{\prime}}$ time is unfortunately wasted because authors fail to take the selection of cases and their writing up sufficiently seriously. This article in the "How to......" series has discussed some of the pitfalls of the writing process and will hopefully reduce wasted effort, especially by clinicians who are inexperienced in writing for publication. Properly done, case reports are an invaluable introduction to medical writing for the inexperienced author and a valuable tool in the hands of the experienced. 